\title{
Fístula bilioduodenal pós colecistite aguda: relato de caso
}

\author{
Biliary tract fistula following acute cholecystitis: case report \\ Marina Araújo Fonte Boa ${ }^{\dagger *}$, Márcio Alexandre Terra Passos ${ }^{\ddagger}$, Marina Pirassol Tepedino ${ }^{\dagger}$, Camila \\ Santos Guimarães ${ }^{\dagger}$
}

Como citar esse artigo. Fonte Boa MA, Passos MAT, Tepedino, MP, Guimarães, CS. Fístula bilio-duodenal pós colecistite aguda: relato de caso. Revista de Saúde. 2016 Jul../Dez.; 07 (2): 31-34

\begin{abstract}
Resumo
Fístulas biliares internas são comunicações estabelecidas entre um segmento biliar e órgãos abdominais, constituindo uma complicação da colecistite. O objetivo desse manuscrito é relatar um caso de fístula bilioduodenal complicada com hemobilia e hemorragia digestiva, descrevendo a conduta adotada. Um paciente masculino, de 75 anos, atendido no Hospital Universitário Sul-Fluminense (HUSF) - Vassouras/RJ foi diagnosticado com colecistite aguda e tratado conservadoramente para regressão inflamatória, diminuindo o risco de lesões biliares durante posterior cirurgia. O caso evoluiu com melena, que foi investigada com endoscopia digestiva alta (EDA), sendo observada úlcera duodenal com drenagem de secreção purulenta; O exame de tomografia computadorizada evidenciou aerobilia, indicativa de fístula bileodigestiva. O tratamento conservador da fístula consistiu de antibioticoterapia devido à remissão da colecistite após a desobstrução pela fístula e devido aos riscos da realização de cirurgia em idoso. É incomum a ocorrência de fístulas biliodigestivas com clínica hemorrágica. O quadro clínico é geralmente inespecífico e o diagnóstico frequentemente atrasado devido à falta de sinais e sintomas patognomônicos.

Palavras-chave: Colecistite; Fístula biliar; Hemobilia.
\end{abstract}

\begin{abstract}
Internal biliary fistulas are established communications between a segment of the biliary tract and abdominal organs as a complication of cholecystitis. This article reports a case of bilioduodenal fistula, complicated with hemobilia and digestive hemorrhage treated conservatively. A male patient with 75 years of age was admitted to Hospital Universitário SulFluminense (HUSF) - Vassouras/RJ and was diagnosed with acute cholecystitis. The patient was treated conservatively aiming at reduction of inflammatory thus decreasing the risk of biliary lesions in a subsequent surgery. The case evolved to melena, which was investigated with a high digestive endoscopy that showed a duodenal ulcer with drainage of purulent secretion. A computed tomography showed aerobilia indicative of bileodigestive fistula. The conservative treatment of the fistula consisted of antibiotic therapy, chosen due the remission of cholecystitis after fistula clearance and due to the risks of surgery in elderly people. Bilodigestive fistulas with haemorrhagicpresentation are uncommon. The clinical features are usually nonspecific and often delay diagnosis due to a lack of pathognomonic signs and symptoms. Keywords: Cholecystitis; Biliary fistula; Hemobilia.
\end{abstract}

\section{Introdução}

As fístulas biliares internas espontâneas são comunicações que se estabelecem entre qualquer segmento da árvore biliar e os órgãos abdominais. Constituem uma afecção rara, apesar de tratarem-se de uma complicação de uma outra patologia muito freqüente da prática médico-cirúrgica, a colecistite. ${ }^{1}$ As fístulas bilioentéricas ocorrem em cerca de 3-5\% dos doentes com colelitíase, sendo $68 \%$ destas, bilioduodenais. ${ }^{2}$

Este trabalho tem como objetivo relatar a presença de fístula bilioduodenal pós colecistite aguda em um paciente que evoluiu com hemorragia digestiva e não foi submetido à correção cirúrgica imediata, e descrever a conduta conservadora adotada, devido às condições clínicas do paciente.

Não é comum na literatura médica, a ocorrência de fístulas biliodigestivas com sinais e sintomas hemorrágicos. O quadro clínico dos casos é geralmente inespecífico e a falta de sinais e sintomas patognomônicos frequentemente atrasa o diagnóstico.

\section{Relato de caso}

O quadro clínico descrito foi autorizado para publicação pelo paciente, através de assinatura em Termo de Consentimento Livre e Esclarecido após devidas orientações sobre a pesquisa a ser realizada.

O paciente em questão, do sexo masculino, 75 anos, sem comorbidades, foi encaminhado para o

Afiliação dos autores: †1Universidade Severino Sombra, Pró-Reitoria de Ciências Médicas, Discente do curso de Medicina

¥ Universidade Severino Sombra, Pró-Reitoria de Ciências Médicas, Docente do curso de Medicina

\footnotetext{
* Endereço para correspondência: Universidade Severino Sombra, Av. Exped. Oswaldo de Almeida Ramos, 280 - Centro - Vassouras,RJ - CEP 27700-000.

E-mail: marina.fonteboa@gmail.com
} 
Hospital Universitário Sul-Fluminense (HUSF), com diagnóstico sindrômico de abdome agudo inflamatório por colecistite aguda. O mesmo queixava-se de dor em cólica no hipocôndrio direito há cerca de cinco dias, associada a vômitos, febre com calafrios e inapetência, porém no momento da admissão relatava melhora da dor em relação aos dias anteriores.

Trazia consigo exame ultrassonográfico (USG) de abdome realizado no mesmo dia em seu município, o qual evidenciava a vesícula biliar distendida e com paredes espessas, compatível com colecistite aguda. Porém não foi visualizado cálculo, e sim lama biliar. Portava também o resultado de um exame de endoscopia digestiva alta (EDA) diagnosticando esofagite Grau A de Los Angeles, hérnia de hiato e gastrite enantematosa.

Na avaliação da admissão, o paciente apresentavase desidratado, discretamente hipocorado, anictérico, acianótico, eupneico em ar ambiente, taquicárdico e normotenso. Abdome doloroso à palpação profunda em hipocôndrio direito, com Sinal de Murphy positivo, ruídos hidroaéreos presentes e normais, sem sinal de irritação peritoneal.

Os exames complementares realizados no HUSF mostraram: hematócrito: $39 \%$; hemácias: 4,51 milhões $/ \mathrm{mm}^{3}$; hemoglobina: $13,5 \mathrm{~g} / \mathrm{dl}$; leucócitos: $16.210 \mathrm{cel} / \mathrm{mm}^{3} \mathrm{com}$ desvio para esquerda (bastões: $9 \%$ ), transaminases normais, fosfatase alcalina normal: $172 \mathrm{U} / 1$, pequeno aumento das bilirrubinas (total: $1,5 \mathrm{mg} / \mathrm{dl}$, direta: $1,0 \mathrm{mg} / \mathrm{dl}$, indireta: $0,5 \mathrm{mg} /$ dl) e amilase normal.

Optou-se por conduta inicialmente conservadora com o objetivo de remissão do processo inflamatório agudo, sendo o paciente internado na enfermaria da clínica cirúrgica do HUSF com prescrição de fluidoterapia, antibioticoterapia com cefazolina e analgesia.

No quinto dia de internação o paciente apresentou quadro de melena sem manifestações hemodinâmicas significativas, com exames laboratoriais evidenciando anemia aguda (hematócrito: $29 \%$; hemoglobina: 10,0 g/dl), sendo realizada a reposição volêmica com fluido cristalóide e dois concentrados de hemácias. Uma nova EDA demonstrou úlcera duodenal profunda de bordas planas com sinais de sangramento recente (H1 de Sakita, Forrest 1lB).

No decorrer dos dias o paciente apresentou piora clínica e laboratorial (hematócrito: $20 \%$; hemácias: 2,30 milhões $/ \mathrm{mm}^{3}$; hemoglobina: $6,8 \mathrm{~g} / \mathrm{dl}$, leucócitos: $16.850 \mathrm{cel} / \mathrm{mm}^{3}$, bastões: $25 \%$, segmentados: $70 \%$, linfócitos: $4 \%$; monócitos: $1 \%$; plaquetas: $145 \mathrm{mil} /$ $\mathrm{mm}^{3}$, uréia: $81 \mathrm{mg} / \mathrm{dl}$, creatinina: $1,5 \mathrm{mg} / \mathrm{dl}$ ), sendo realizada nova transfusão de concentrado de hemácias e transferência para a enfermaria da clínica médica do HUSF três dias após a piora.

No décimo quarto dia de internação o paciente continuava a apresentar melena e episódios de hipotensão, sendo realizada nova reposição de concentrado de hemácias. Nesta oportunidade o hematócrito encontrava-se em $22 \%$.

No décimo sexto dia de internação foi realizada nova EDA para reavaliação do sangramento sendo evidenciado úlcera duodenal bulbar de parede anterior profunda com drenagem de secreção purulenta pela sua luz, sendo aventada a possibilidade de tratar-se

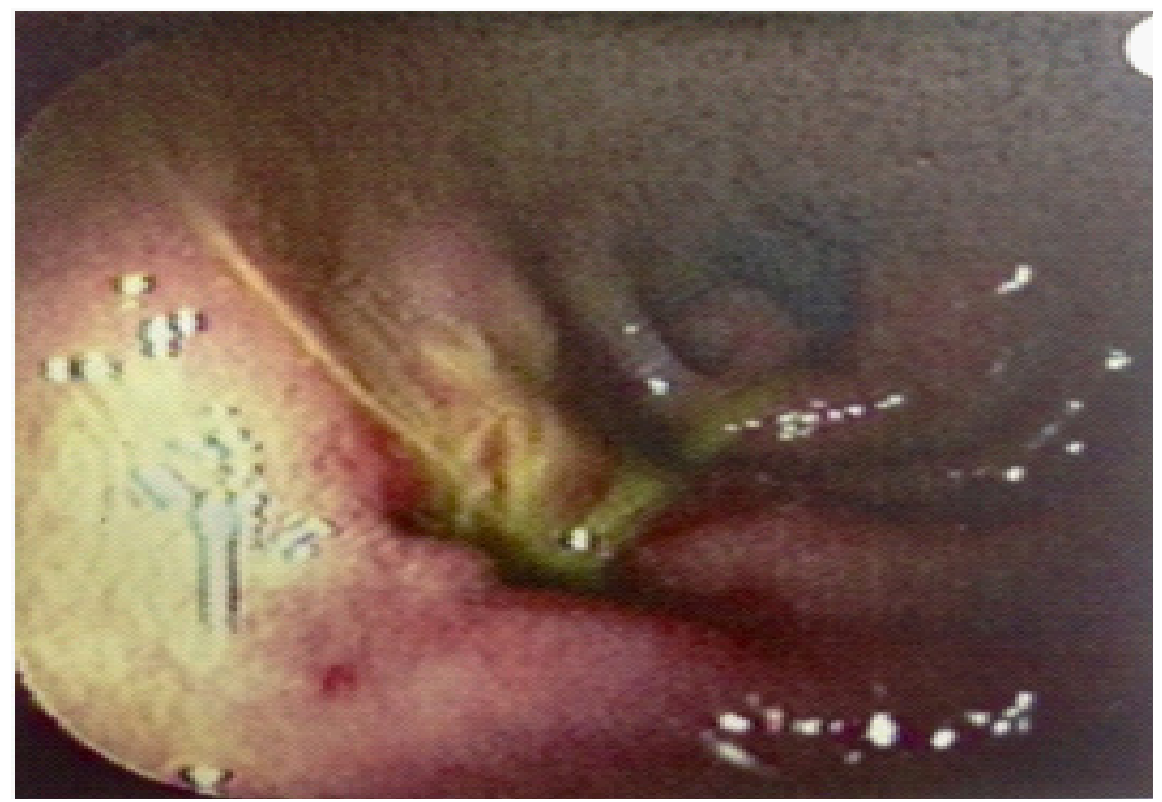

Figura 1. Úlcera duodenal bulbar de parede anterior possivelmente perfurada e tamponada parcialmente com drenagem de abscesso perivisceral para lúmen duodenal. Imagem cedida por Dr. Márcio Terra Passos. 
de úlcera perfurada e tamponada parcialmente, com drenagem de abscesso perivisceral para lúmen duodenal (Figura 1). A radiografia de tórax não demonstrou sinais característicos de pneumoperitônio. Diante destes resultados foi iniciado ceftazidima e metronidazol, e suspensa a dieta via oral, com tubagem gástrica por sonda nasogástrica em sifonagem.

Nos dias subsequentes paciente continuou a apresentar melena, mantendo-se estável hemodinamicamente. Exames laboratoriais mostrando elevação do hematócrito ( $28 \%$ ) e do número de plaquetas (135 mil $/ \mathrm{mm}^{3}$,), e queda na contagem de linfócitos $\left(4.230 \mathrm{cel} / \mathrm{mm}^{3}\right)$, neutrófilos segmentados $(75 \%)$ e bastões (1\%), e demais exames dentro da normalidade. Assim sendo, foi retirada a sonda nasogástrica (débito de $100 \mathrm{ml}$ em 12 horas) e reiniciada dieta oral.

No décimo segundo dia de internação o paciente não mais apresentou melena e subsequentemente foram realizada uma nova EDA e uma tomografia computadorizada (TC) de abdome com contraste para

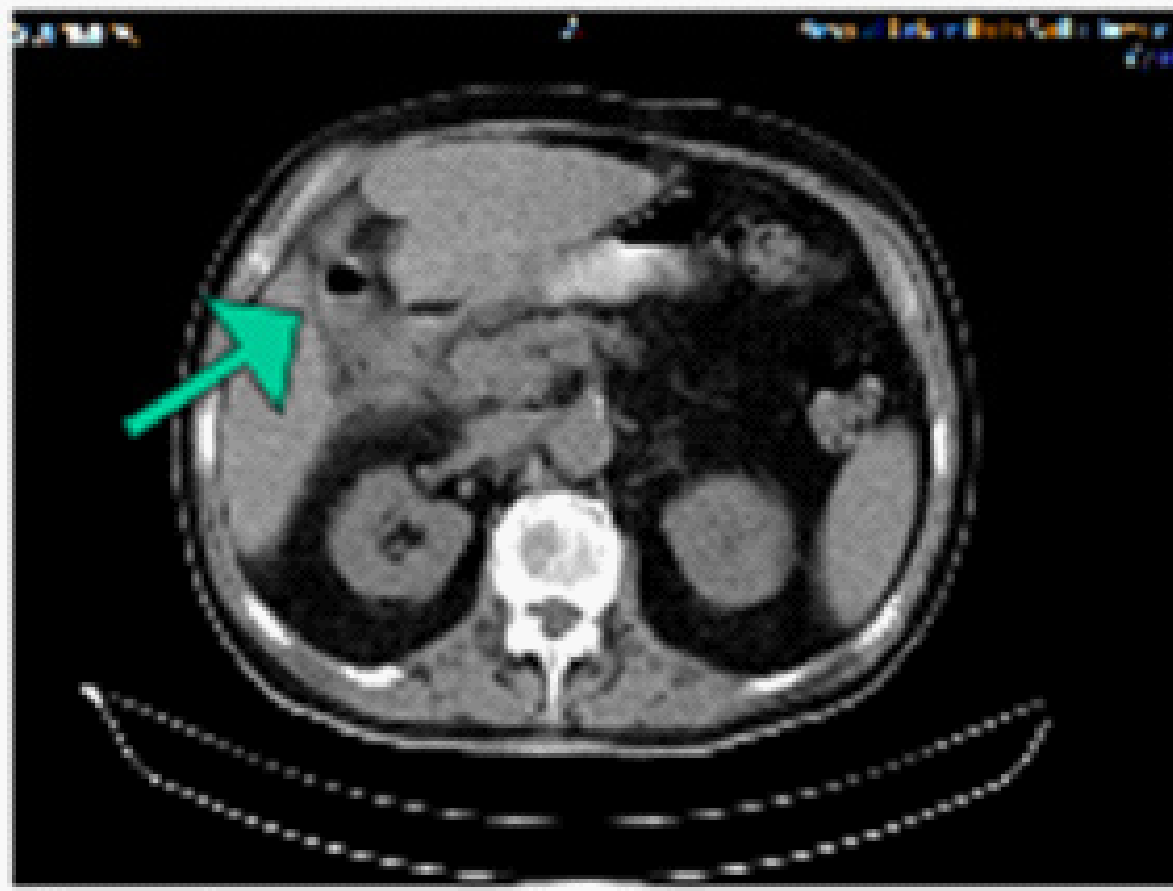

Figura 2. Tomografia computadorizada de abdome evidenciando gás nas vias biliares intrahepáticas (aerobilia), indicativo de presença de fistula bileodigestiva. Imagem cedida pelo Dr. Márcio Terra Passos.

acompanhamento do caso.

A EDA mostrava lesão profunda na parede anterior do bulbo duodenal com confluência de pregas compatíveis com úlcera péptica perfurada e tamponada ou fistulizada. A biópsia antral para análise histopatológica e pesquisa direta de $\mathrm{H}$. pylori não revelou alterações. A TC de abdome evidenciou presença de gás nas vias biliares intra-hepáticas indicando presença de fistula bileodigestiva (Figura 2).

Com a continuidade da antibioticoterapia até completar-se dez dias de tratamento, um quadro clínico estável, bom estado geral com estabilidade hemodinâmica e ausência de intercorrências, o paciente recebeu alta hospitalar com segmento ambulatorial sendo mantido omeprazol e bromoprida.

O último exame do paciente, dois dias antes da alta apresentou hematócrito: $32 \%$; hemácias: 3,73 milhões $/ \mathrm{mm}^{3}$; hemoglobina: 10,6 g/d1, leucócitos: 3.630 cel $/ \mathrm{mm}^{3}$, bastões: $3 \%$, segmentados: $60 \%$, linfócitos:
$29 \%$; monócitos: $1 \%$; plaquetas: $189 \mathrm{mil} / \mathrm{mm}^{3}$, uréia: $18 \mathrm{mg} / \mathrm{dl}$, creatinina: $1,1 \mathrm{mg} / \mathrm{dl}$ ).

\section{Discussão}

A fístula biliodigestiva constitui uma afecção rara apesar de tratar-se de complicação de uma patologia muito freqüente da prática médico-cirúrgica, a colecistite. ${ }^{1}$ No quadro clínico descrito, o tipo de fístula biliodigestiva encontrada foi a bilioduodenal, esta que é considerada a mais prevalente em $68 \%$ dos casos, seguida pelas fístulas bilioentéricas, sendo a biliocolônica (5-25 \%) e coledococolônica $(2,5 \%)$ as menos comuns. ${ }^{2}$

A principal etiologia das fístulas biliodigestivas é a colelitíase crônica recorrente, sendo que entre outras causas podemos citar a perfuração de uma úlcera duodenal para a árvore biliar, e uma infiltração 
neoplásica a partir da árvore biliar ou no trato gastrointestinal. ${ }^{3}$ Com a evolução das técnicas de intervenção cirúrgica e endoscópica sobre as estruturas hepatobiliares, as fístulas biliodigestivas são hoje, muito frequentemente, resultado de complicações de tais intervenções. ${ }^{4}$ Sua incidência tem sido relatada em cerca de 3-5\% dos pacientes com colelitíase e em $0,15-4,8 \%$ de todos os que se submetem a operações nas vias biliares. ${ }^{5}$ Neste relato de caso, a etiologia identificada para a ocorrência da fístula foi o episódio de colecistite aguda apresentada pelo paciente, que negou qualquer episódio anterior de cólica biliar.

Na colecistite aguda, o ducto cístico é obstruído por edema ou cálculos, o que gradualmente leva a inflamação grave da vesícula biliar. Em conseqüência disso, há diminuição do fluxo sanguíneo para o órgão, causando isquemia, necrose e por fim a perfuração da vesícula para algum órgão oco adjacente ou peritônio. A perfuração da vesícula biliar como uma complicação da colecistite aguda foi descrita pela primeira vez por Courvoisier em $1890 .^{6}$

O paciente do caso descrito, apresentou uma forma clínica incomum de fístulas biliodigestivas. Os sinais e sintomas hemorrágicos não sugeriam a presença de fistula, como é com frequência reportado na literatura. ${ }^{4} \mathrm{~A}$ sua expressão foi a mais temível das complicações: a hemobilia associada à hemorragia digestiva, podendo esta também estar relacionada com a úlcera duodenal encontrada em uma das EDA realizadas durante a internação. As complicações observadas tornaram o caso mais complicado, colocando em rsico a vida do paciente pela dificuldade inicial em se esclarecer a origem do sangramento e pelos episódios hemorrágicos recorrentes que o mesmo experimentou.

O diagnóstico de fístulas biliodigestiva é frequentemente atrasado por causa da falta de sinais e sintomas patognomônicos, levando a um aumento da mortalidade e morbilidade.

A colecistite aguda é uma patologia comum na prática médica de indicação eminentemente cirúrgica, alguns autores defendem seu tratamento não cirúrgico inicial e posterior abordagem operatória com intuito de regressão da reação inflamatória peri-colecística, diminuindo o risco de lesões iatrogências da árvore biliar. $^{6}$

A decisão por um tratamento conservador da fístula consistindo de antibioticoterapia e reposição volêmica foi realizada devido à remissão do quadro de colecistite aguda pós desobstrução da fístula, e também devido à hemorragia, que apesar de volumosa cessou espontaneamente, além do risco da realização do procedimento cirúrgico em paciente idoso com intensa resposta inflamatória sistêmica.

Classicamente, as fístulas biliodigestivas, independentemente da presença ou ausência de sintomas, tem sido tratada com ressecção da fístula com colecistectomia, e se necessário exploração das vias biliares. ${ }^{7}$

Esta máxima cirúrgica tem sido questionada nos últimos anos. Vários grupos têm defendido a observação do paciente assintomático, reservando a cirurgia para aqueles pacientes que apresentam sepse biliar. Estes autores argumentaram que, dada a expectativa de vida e comorbidades médicas de alguns pacientes, a cirurgia como uma medida terapêutica e/ou preventiva seria de benefício limitado. Embora essa política seja defendida em um número limitado de relatos de casos, parece uma abordagem válida.?

O presente estudo teve relevância bibliográfica por concordar com a literatura clínico-cirúrgica atual ao demonstrar que o tratamento conservador pode ser de grande valia em pacientes com fístula biliodigestiva. Cabe ressaltar que as condutas médicas devem sempre ser individualizadas e direcionadas à condição clínica apresentada por cada paciente.

\section{Referências}

1.Machado MAC, et al. Fístulas biliares internas: estudo de 13 casos e revisão de literatura. Ver.Hosp.Clín Fac Med S Paulo, 1995; 50(1): 45-48.

2. Lee SB, et al. Acute acalculous cholecystitis associated with cholecystoduodenal fistula and duodenal bleeding. A case report. Korean J Intern Med, 2003; 18(2): 109-114.

3. Stagnitti F, et al. Biliodigestive fistulae and gallstone ileus: Diagnostic and therapeutic considerations. Our experience. G Chir, 2014; 35(9-10): $235-238$

4. Jorge JX, et al. Fístulasbiliodigetsivaslítiasicas (a proposito de 3 casosclínicos). GE J Port Gastrenterol, 2013; 20(3):119-122

5. Angrisani L, et al. Cholecystoenteric fistula is not a contraindication for laparoscopic surgery. Surg Endosc, 2001; 15: 1038-1041

6. McGillicuddy, et al. Non-operative management of acute cholecystitis in the elderly. Bristisb Journal of Surgery, 2012; 99: 1254-1261

7. Balent E, et al. Cholecystocolonic fistula. Hawaii J Med Public Health, 2012; 71(6): 155-157 